\title{
Uma Análise Generificada de Cuidadoras de Crianças com Desenvolvimento Atípico
}

\author{
Un Análisis Generalizado de Cuidadoras de Niños con Desarrollo Atípico
}

A Generalized Analysis of Caregivers of Children with Atypical Development

\author{
Matheus dos Santos da Silveira \\ ORCID: https://orcid.org/0000-0003-4724-4443 \\ Universidade Federal do Pará, Pará/Brasil \\ Yasmin Borges Farias \\ ORCID: https://orcid.org/0000-0002-1566-8877 \\ Universidade Federal do Pará, Pará/Brasil
}

Ruth Daisy Capistrano de Souza ORCID: https://orcid.org/0000-0001-5911-0261 Universidade Federal do Pará, Pará/Brasil

Declaração de Direito Autoral

A submissão de originais para este periódico implica na transferência, pelos autores, dos direitos de publicação impressa e digital. Os direitos autorais para os artigos publicados são do autor, com direitos do periódico sobre a primeira publicação. Os autores somente poderão utilizar os mesmos resultados em outras publicações indicando claramente este periódico como o meio da publicação original. Em virtude de sermos um periódico de acesso aberto, permite-se o uso gratuito dos artigos em aplicações educacionais e científicas desde que citada a fonte conforme a licença CC-BY da Creative Commons.

\section{Resumo}

O desenvolvimento humano é um fenômeno multifacetado, envolvendo processos de mudanças biológicas, psicológicas, sociais e culturais, ocorridos nas interações entre pessoas e sistemas biológicos, dentro de grupos sociais e ambientes, ao longo do ciclo da vida. O sistema familiar é visto como um dos ambientes centrais para a pessoa em desenvolvimento, dado o estabelecimento de relações entre a criança, desde o início de sua vida, e seus cuidadores. O objetivo deste estudo foi analisar, a partir dos estudos de gênero e do modelo bioecológico do desenvolvimento humano, os papéis desempenhados por cuidadoras de crianças com desenvolvimento atípico, no contexto da Amazônia brasileira. Os estudos sobre o cuidado de crianças com desenvolvimento atípico mostram o quanto esse fator age como um divisor no exercício da parentalidade, dados os aspectos socioculturais de gênero, raça e classe, onde se percebeu a maior parte das pessoas que exercem tais atividades, sendo mulheres. Concluiu-se que as práticas de cuidado ainda são atravessadas por toda uma carga de ações e respostas de uma rede de contextos cujas atividades, continuam sendo vistas como "domínios" femininos.

Palavras-chave: Desenvolvimento humano; Análise generificada; Cuidadoras; Crianças com desenvolvimento atípico.

\section{Resumen}

El desarrollo humano es un fenómeno multifacético, involucrando procesos de cambios biológicos, psicológicos, sociales e culturales, ocurridos en las interacciones entre personas y sistemas biológicos, dentro de grupos sociales y ambientes, a largo del ciclo vital. El sistema familiar es visto como uno de los entornos centrales para la persona en desarrollo, dado el establecimiento de 
relaciones entre el niño, desde el principio de su vida, y sus cuidadores. El objetivo de este estudio fue analizar, a partir de los estudios de género y el modelo bioecológico del desarrollo humano, los roles desempeñados por los cuidadores de niños con desarrollo atípico, en el contexto de la Amazonía brasileña. Los estudios sobre el cuidado de niños con desarrollo atípico muestran cómo este factor actúa como un factor divisorio en el ejercicio de la paternidad, dados los aspectos socioculturales del género, la raza y la clase, donde se encontró que la mayoría de las personas que realizan tales actividades son mujeres. Se llegó a la conclusión de que las prácticas de cuidado todavía están atravesadas por una gran cantidad de acciones y respuestas de una red de contextos cuyas actividades continúan siendo vistas como "dominios" femeninos.

Palabras clave: Desarrollo humano; Análisis generificada; Cuidadores; Niños con desarrollo atípico.

\begin{abstract}
Human development is a multifaceted phenomenon, involving processes of biological, psychological, social and cultural changes, occurring in the interactions between people and biological systems, within social groups and environments, throughout the life cycle. The family system is seen as one of the central environments for the developing person, given the establishment of relationships between the child, from the beginning of his life, and his caregivers. The objective of this study was to analyze, from the gender studies and the bioecological model of human development, the roles played by the caregivers of children with atypical development, in the context of the Brazilian Amazon. Studies on the care of children with atypical development show how this factor acts as a divisive factor in the exercise of paternity, given the sociocultural aspects of gender, race and class, where it was found that most people who perform such activities are women. It was concluded that care practices are still traversed by a large number of actions and responses from a network of contexts whose activities continue to be seen as feminine "domains".
\end{abstract}

Keywords: Human development; Gendered analysis; Caregivers; Children with atypical development.

\section{Introdução}

O desenvolvimento pode ser visto, de acordo com Senna e Dessen (2016), como um fenômeno multifacetado, composto pelo conjunto de processos de mudanças progressivas, estruturais e organizacionais, ocorridos nas interações entre pessoas e sistemas biológicos, dentro de grupos sociais e ambientes, no decorrer do tempo (Cairns, Elder, \& Costello, 1996; Magnusson \& Cairns, 1996). Portanto, as buscas de esclarecimentos e ou mesmo a organização e levantamento de dados que possam contribuir para as formações humanas num determinado sistema são importantes, para a construção de conhecimentos relacionados à área e a fomentação de novas políticas públicas, mais sensíveis à realidade (Bronfenbrenner, 1989).
Dessen e Guedea (2005) compreendem o funcionamento deste sistema, faz-se necessário investigar os contextos, as propriedades estruturais e funcionais da pessoa e do ambiente, e como eles interagem e produzem constâncias e mudanças no desenvolvimento do indivíduo. Portanto, essa afirmativa, corrobora ao que Bronfenbrenner (2011) apresenta em sua teoria, dentre seus pressupostos (modelo PPCT) como um de seus fundamentos, o desenvolvimento humano, num processo de interrelação em seu meio, ou seja, as relações com seu ambiente.

A bioecologia do desenvolvimento humano, enquanto perspectiva teórica foi construída a partir das contribuições de autores como Bronfenbrenner (1989, 2011). Essa teoria tem em vista o entendimento da pessoa como ser ativo e constituinte de uma série de contextos, com ambientes que possuem 
propriedades capazes, ou não, de contribuir para o pleno desenvolvimento da pessoa.

Os contextos englobam qualquer acontecimento ou condição exterior ao organismo (Lemos \& Veríssimo, 2015) são vistos como um dos eixos primordiais para a compreensão do desenvolvimento, sendo parte essencial das proposições de Bronfenbrenner (1989), em especial na década de 1990. Dentre os diversos contextos dos quais uma pessoa encontra-se inserida ao longo de sua vida, o mais próximo a ela é o microssistema, ambiente no qual as relações pessoa-pessoa ocorrem, considerando os processos de observação realizados por uma pessoa, seu engajamento em atividades relacionais $\mathrm{e}$ gradativamente mais complexas, que contribui para o desenvolvimento de recursos, e consequentemente, para o desenvolvimento mais pleno, completo.

Ao longo de suas obras, Bronfenbrenner (2011) apresenta o microssistema familiar enquanto um dos ambientes centrais para a pessoa em contexto, dadas as relações que são estabelecidas, por exemplo, entre criança (desde o início de sua vida) com seu cuidador. A presença do cuidador, ou seja, àquele que dispensa à outra pessoa (como a criança) o seu tempo, recursos e habilidades voltadas para o exercício do cuidar, parece resultar no fortalecimento de suas relações. Assim, a prática desse cuidar, passa a ser figura chave para o entendimento do desenvolvimento, em estágios iniciais do ciclo vital; destarte, o estudo dos cuidadores que vem sendo tema central em diversos estudos da perspectiva bioecológica do desenvolvimento humano nos últimos anos.

O exercício da parentalidade, ou seja, de práticas relativas à promoção do desenvolvimento físico, psicossocial, emocional e cognitivo das crianças por parte dos seus cuidadores, sofre significativa influência de aspectos do ambiente, cujas famílias se encontram inseridas, como Câmara et al. (2016) afirmam que a relação entre o ato de cuidar e o cuidador é determinada por fatores pessoais dos indivíduos envolvidos, da estrutura familiar existente e do contexto no qual se inserem a nível cultural, social, político e econômico. Corroboram, ainda, ao mencionar que "a depender do contexto, o cuidar torna-se desafiador ou de difícil execução, influenciado pelas dificuldades vivenciadas por ocasião da doença que somadas à responsabilidade do cuidador, pode desencadear uma sobrecarga de atribuições" (p. 270).

Nesse sentido, é importante investigar quem está desempenhando tais práticas de cuidado. Nota-se a partir de estudos (Morris, 2001; Swinkels, van Tilburg, Verbakel \& van Groenou, 2017) a predominância de mulheres no exercício dessa função, fato esse que deve ser problematizado, principalmente em uma análise regional. $O$ que de fato leva a um número expressivamente maior de mulheres desempenharem atividades vinculadas ao cuidado, comparado a homens? Para responder esse questionamento, considerando a realidade amazônica na qual os pesquisadores estão inseridos, a presente investigação possui como objetivo analisar, a partir dos estudos de gênero e do modelo bioecológico do desenvolvimento humano, os papéis desempenhados por cuidadoras, sendo feito um recorte às cuidadoras de crianças com desenvolvimento atípico.

\section{A “pessoa" na teoria bioecológica}

O desenvolvimento humano, a partir da proposta de Bronfenbrenner (2011), é entendido enquanto um conjunto de continuidades e descontinuidades, sendo, dessa forma, um processo, no qual se evidenciam características biopsicológicas das pessoas, dispostas ao longo do ciclo vital, atravessando gerações em um tempo histórico, tanto passado quanto presente e futuro. $\mathrm{O}$ foco da teoria é visto na interação entre a pessoa e o ambiente, considerando as influências, que são bidirecionais.

Uma vez objetivando-se abordar a figura das cuidadoras dentro de uma perspectiva bioecológica, faz-se necessário descrever os aspectos que influenciam o 
desenvolvimento acerca da pessoa. Dentro do modelo PPCT (processo-pessoa-contextotempo), Poletto e Koller (2008) definem que as características da pessoa são tidas como produtoras e produtos de seu desenvolvimento ao influenciarem diversamente nos processos proximais, ou seja, as interações do sujeito em desenvolvimento com o outro, também em desenvolvimento. Bronfenbrenner e Morris (2006) discutem que existem três características biopsicossociais da pessoa que moldam o seu desenvolvimento: as disposições, os recursos biológicos e as características de demanda.

Carvalho-Barreto (2016) afirma que as disposições são o conjunto de habilidade das pessoas se engajarem ativamente em atividades, seja individualmente ou com outras pessoas; tal afirmação é ratificada por Bronfenbrenner e Morris (2006), ao postularem que estas podem ativar os processos proximais em direção ao desenvolvimento. As características de recurso, por sua vez, são as necessidades que uma pessoa tem para atingir seu desenvolvimento pleno, em determinado estágio do desenvolvimento (CarvalhoBarreto, 2016; Bronfenbrenner \& Morris, 2006). Por fim, as demandas são características pessoais que podem facilitar ou dificultar as relações no contexto social.

Bronfenbrenner e Morris (2006) ressaltam que este componente é de suma importância para a compreensão do contexto em que esta se dá o desenvolvimento. Os autores ressaltam que essas três características da pessoa se incorporam na definição de microssistema, a pessoa como biopsicossocial, como as características de toda uma rede de apoio e de pessoas que participam da vida da pessoa em desenvolvimento, como as características dos cuidadores na vida da criança com deficiência e vice-versa (Bronfenbrenner, 2011). Em uma compreensão mais abrangente, serão a partir destes três componentes que a pessoa irá moldar seus ambientes, evocar respostas deles e reagir a eles (Darling, 2007).
A partir desse aporte teórico, discute-se que embora as contribuições bioecológicas favoreçam discutir à realidade a qual estamos inseridos de uma forma mais complexa, ela por si não é suficiente para fundamentar uma análise generificada de cuidadoras de crianças com desenvolvimento atípico. Isso se dá pelo fato de que o constructo gênero aparece de forma coadjuvante nos trabalhos do autor, como apresenta Darling (2007), ao discutir como a compreensão de Bronfenbrenner acerca dos processos de socialização de garotas e garotos resultou em diferentes padrões de comportamentos diferentes, e logo, em processos desenvolvimentais distintos.

Para além da perspectiva bioecológica, mas ainda sobre a ótica da pessoa, os estudos sobre gênero se apresentam, nesse sentido, enquanto uma rica ferramenta a esta discussão, tendo em vista que papéis construídos e reforçados pela sociedade ocidental contemporânea acerca dos papéis a serem desempenhados por cuidadoras, ainda se enquadram em um modelo assimétrico de poder, patriarcal e centralizado na figura do cuidar. De acordo com Butler (2003), o gênero é performativo, produzido através de modos de agir. Connell (2009) ratifica essa afirmação, ao discutir como o constructo "gênero" é apresentado como natural à realidade social, quando na verdade ele deve ser entendido enquanto um processo construído a partir das interações sociais.

Autores como Monteiro (2001) e Wagner, Prebedon, Mosmann \& Verza (2005) apontam os papéis de gênero como partes estruturantes das relações familiares, em que os homens possuem o "dever" de prover financeira e materialmente, enquanto que as mulheres possuem o "dever" de cuidar da habitação e dos filhos. A existência de novos arranjos de estruturas familiares deve ser destacada, tendo em vista sua problematização frente à máxima apresentada em relação aos papéis de gênero (Henriques, Féres-Carneiro \& Magalhães, 2006).

Para Silva, Pontes, Lima \& Maluschke (2010), o contexto sociocultural é 
preponderante para analisar as funções que vêm sendo desempenhadas por membros de um microssistema familiar, considerando o ambiente como lócus para a atribuição de papéis específicos para homens e para mulheres, construindo dessa forma, uma heterogeneidade nas atividades, e logo, nas interações realizadas. Logo, faz-se necessário debater acerca dos constructos gênero e classe, partindo do pressuposto de que ambos são categorias de análise para os estudos do desenvolvimento humano.

Os estudos apresentados apontam a necessidade da discussão acerca das atividades, dos papéis e das relações desempenhados pelas pessoas nos processos de desenvolvimento. Os valores, construídos e reforçados no macrossistema, acabam por influenciar na constituição do que é socialmente entendido enquanto válido (Bronfenbrenner, 1989), como, por exemplo, os valores imbricados no cuidado de crianças. A pessoa desenvolvente, nesse caso a mulher, além de ser atravessada por todas as questões do gênero, se vê em face das expectativas de papéis que são cobradas pelo social, não só nas relações que envolvem o cuidado, mas tudo aquilo que diz respeito à criança com desenvolvimento atípico. Daí a importância de se analisar categorias como gênero e outras que se relacionam a esta.

\section{Gênero e classe como categorias analíticas}

Ao refletir acerca das cuidadoras de crianças com desenvolvimento atípico, é necessário tecer considerações acerca das discussões de gênero e classe enquanto categorias analíticas, ainda mais considerando a realidade presenciada no Brasil. Para tal, parte-se do pressuposto tomado pelos estudos interseccionais, que afirmam que, para analisar de forma holística as relações sociais nas quais as mulheres participam, as múltiplas formas de identidades experienciadas devem ser consideradas, sendo essas, gênero, raça e classe as mais trabalhadas (Kerner, 2012; Hirata, 2014). Portanto, analisar um fenômeno de forma interseccional, aponta para se buscar conhecer de forma mais abrangente, a realidade das relações sociais que está sendo construída em nosso ambiente, objetivando tornar o conhecimento mais complexo, logo, mais próximo da realidade, uma "arma política" para a luta contra as opressões existentes (Collins, 2014).

O trabalho de Hirata (2014) é de rica contribuição para as discussões acerca do cuidado, sob um viés interseccional. A autora pontua que, a partir de uma análise comparativa entre cuidadoras brasileiras, francesas e japonesas, o maior estrato é de mulheres pertencentes a classes socioeconômicas mais desfavorecidas. Embora, ressalvas devam ser feitas (as participantes do estudo eram cuidadoras formais, remuneradas, e não informais, objeto do presente estudo), os dados apresentados mostram quais papéis estão postos culturalmente, sendo reforçados.

Charmes (2005) ratifica essa afirmação, ao afirmar que, em média, mulheres gastam mais tempo de sua rotina em atividades domésticas e não pagas do que homens. Em um cenário brasileiro, as reflexões que Pinto (2013) levanta no que diz respeito ao fato de que mulheres que se encontram em camadas socioeconômicas mais vulneráveis e que, muitas vezes, necessitam realizar uma dupla jornada entre o trabalho pago e o trabalho doméstico, a concorrência entre buscar meios de sustentar sua família, ao mesmo tempo em que deve manter as práticas de cuidado à seus filhos, refletem as condições que muitas cuidadoras vivenciam, o que deve ser problematizado a fim de buscar melhores condições para essa população. Além disso, muitas vezes o acesso ao trabalho é informal e precarizado, principalmente no contexto brasileiro e latino-americano, o que reforça a necessidade de estudos na área (Souza \& Mariano, 2018).

Nesse sentido, é importante verificar como os papéis exercidos pelas cuidadoras estão atravessados por marcadores de gênero e classe, pois se reafirma o conceito de mulher como uma categoria plural e diversa de contextos em que esses sujeitos estão 
inseridos, discutindo-se as questões da invisibilidade e das relações de poder em uma realidade como a brasileira, em contexto de exclusão e subalternidade (Furiati \& Mayorga, 2016).

De acordo com Wagner et al., (2005) com as mudanças sociais e a entrada das mulheres no mercado de trabalho e o advento do movimento feminista, as famílias em geral adquirem um novo perfil quanto aos seus integrantes, em contraponto ao modelo da família tradicional, nuclear, onde o pai é o provedor, e a mãe, cuidadora. Souza e Mariano (2018) destacam, em pesquisa realizada com 190 beneficiárias do Programa Bolsa Família (PBF) de Curitiba e Fortaleza, entre os anos de 2013 e 2014, no CRAS (Centros de Referência de Assistência Social); a perspectiva geracional, ou seja, a passagem de valores e conhecimentos de mãe para filha, onde pode perceber-se essa mudança de percepções e comportamentos, sobretudo sobre a divisão sexual do trabalho e nas atividades do cuidado, reconhecendo-se o acesso a educação formal como um dos principais mediadores dessa transformação. Sorj (2013) também destaca como mediadores o acesso aos meios de comunicação, aos direitos humanos e a disseminação da perspectiva feminista.

Assim posto, vê-se que os diversos contextos vividos pelas mulheres acabam por criar e ou mesmo, dar relevância aos aspectos das subjetividades que se traduzem em lutas, posições políticas, opressões, reproduções de poder (Sorj, 2014; Furiati \& Mayorga, 2016). Os autores encontraram em sua pesquisa sobre as vozes das mulheres que além de outros fatores, a precarização econômica afeta seus padrões de vida, onde se gera efeitos que vão da exclusão, invisibilidade a outras formas de opressão.

A interseccionalidade de características minoritárias como as relações de gênero e pobreza nas situações que perpassam as mulheres, é destacada por Miranda (2016), quanto à produção e intensificação das contingências de vulnerabilidade dos indivíduos em questão. Sorj (2013) destaca que a questão da renda influencia diretamente na experiência doméstica das mulheres, e que a participação masculina na divisão sexual do trabalho doméstico, como fato relevante de cuidado, permanece, até certo ponto, ausente das discussões, de tal forma que a questão do "care" aparece como um problema de e para as mulheres.

$\mathrm{O}$ que de fato se constata na realidade social, mesmo nos dias atuais, a mulher, ainda têm papel minoritário (ocupações domésticas e de cuidado, se sobrepõem) se comparado ao destaque que se dá ao homem (trabalho fora da esfera do ambiente doméstico), o que ainda trás em seu bojo, os resquícios de um processo histórico, com longas e fortes evidências de um modelo patriarcal.

\section{Reflexões sobre o ato de cuidar}

O fenômeno do cuidar é um campo rico de estudos para áreas como a Psicologia do Desenvolvimento, devido a sua multidimensionalidade. Sorj (2013) entende o cuidado, seja na esfera privada do seio familiar ou fora dele, como atividades relativas a afazeres domésticos oferecidos a pessoas que poderiam realizá-los por elas mesmas e atividades para assistir pessoas dependentes. Os estudos sobre o cuidado buscam sistematizar os diferentes tipos de atos que são dispensados às pessoas, como Mthembu, Brown, Cupido, Razack \& Wassung (2016), que conceituaram cuidador familiar enquanto alguém que provê cuidado físico e emocional não pago para uma pessoa doente ou deficiente em casa, que pode incluir pais, crianças, cônjuges, assim com vizinhos e amigos. Mas, para Silva, Barros e Hora (2011), afirma que existe a diferença existente entre cuidadores familiares primários $\mathrm{e}$ secundários, sendo os primeiros aqueles que estão mais presentes na rotina das pessoas cuidadas.

Tradicionalmente, o cuidado é vinculado à figura feminina, principalmente em famílias de baixa renda, quando há presença de crianças pequenas. O trabalho 
destinado ao cuidado de pessoas e afazeres domésticos muitas vezes impede a participação feminina no trabalho formal (Souza \& Mariano, 2018), refletindo a construção social dos papéis de gênero presente na realidade brasileira e nortista, ainda sob uma lógica dicotômica de atividades públicas e privadas (Okin, 2008). Segundo os autores, a prevalência das mulheres no que concerne ao trabalho doméstico, cuidado de pessoas e uso do tempo contribui para uma visão de que estas seriam meio, ou seja, uma ferramenta para atender as necessidades do outro.

A experiência de cuidar de outra pessoa pode gerar consequências positivas e negativas para quem as realiza (Kahana \& Young, 1990; Oliveira et al., 2008). No que tange às consequências positivas, o cuidado, ademais, pode resultar em consequências negativas para a saúde daquelas que dispensam tais atividades, em domínios como a saúde física, psicológica, emocional e espiritual (Acton, 2002). Entretanto, de acordo com Poletto e Koller (2008), o ato do cuidado pode ser tido como um fator de proteção, que ao serem abordados como processos proximais, tem potencialidade de alteração na trajetória de vida da pessoa.

Essa multiplicidade nas consequências é descrita no paradigma do cuidar, nos termos de Kahana e Young (1990). O cuidar, para as autoras, deve ser entendido, e logo estudado, para além da unidirecionalidade da interação (cuidador desempenha atividade A para a pessoa cuidada), pois, dessa forma, não é levado em consideração conceitos como flexibilidade, diversidade e multidimensionalidade, centrais para o estudo dos fenômenos do desenvolvimento humano. Nesse sentido, Kahana e Young (1990) desenvolveram uma série de modelos explicativos relacionados ao paradigma do cuidar, desde explicações assimétricas (que enfocam apenas um membro da díade cuidadora-cuidado) até explicações relacionais e dinâmicas (em que ambas as partes da díade são levadas em consideração).
Estudar fenômenos e relações que atravessam as práticas realizadas por cuidadoras é um importante tema para os estudos de desenvolvimento humano, pois, em muitos momentos, a saúde desse grupo social é negligenciada, o que, a partir do fato de suas atividades nessa função possam ser desgastantes, acaba por contribuir para a manifestação de fatores de risco para a sua qualidade de vida, e, à luz de uma perspectiva bioecológica, da qualidade de vida de seu microssistema familiar (Sena, Souza \& Andrade, 2016).

\section{O "cuidar" de crianças com alterações no desenvolvimento}

$\mathrm{O}$ ato de cuidar pode ser realizado em diversos momentos da vida, entretanto, essa função relativa ao cuidar pode necessitar de diversas reorganizações, dependendo de fatores endógenos e exógenos ao microssistema, ao exemplo de crianças que possuam alguma alteração no desenvolvimento. Essa transição vem sendo bem documentada na literatura da área da Psicologia do Desenvolvimento, seja na descrição das experiências dessas cuidadoras com um novo cenário (Sena et al., 2016).

As alterações no desenvolvimento considerado típico podem se apresentar em domínios como o físico, neuropsicomotor (ex.: Transtorno do Déficit de Atenção e Hiperatividade), sensorial e comportamental (ex.:), e requerem a aquisição de repertórios específicos por parte dos cuidadores para que estes possam desempenhar tal papel. $\mathrm{O}$ não desenvolvimento esperado da criança, que, para Pontes de Lima e de Oliveira Lima (2012) compreende ao “(...) crescimento físico, passando pela maturação neurológica, comportamental, cognitiva, social e afetiva" (p.12), infere em significativas alterações no ambiente familiar no qual esta se encontra. Franco (2016) contribui com sua afirmação ao mencionar que a detecção de sinais que permitam chegar precocemente a um diagnóstico é, no entanto, fundamental, porque só isso permite fazer intervenção precoce, ou seja, agir cedo no processo de 
desenvolvimento. Portanto, a partir da demanda apresentada, novas estratégias devem ser pensadas para o funcionamento do contexto, a exemplo dos comportamentos de cuidado dispensados à criança por parte dos familiares (Van Keer et al., 2017).

A nova realidade enfrentada acaba por afetar a estrutura familiar, conceito esse que mudou ao longo do tempo, já que este é inerente ao tempo e a cultura, moldando assim suas estruturas. A partir de uma perspectiva desenvolvimental, a família pode ser compreendida como "um sistema no qual é caracterizada por padrões de interação únicos entre seus membros" (Anderson \& Sabateli, 2007 apud Kotzampopoulou, 2015), e esta é afetada tanto positiva quanto negativamente; tal impacto causado nos cuidadores e no microssistema familiar pode variar de acordo com a personalidade da criança, o tipo de deficiência ou comprometimento, o estilo da família, saúde mental, além do bem-estar e atitudes dos cuidadores (Kotzampopoulou, 2015).

Tal fato contribui com o aumento de investigações sobre de aspectos relacionados aos cuidadores de crianças com alterações no desenvolvimento, como afirma Faria (2011), pois, essas pessoas, ao assumirem a função de cuidadores, apresentam sentimentos como sobrecarga, medo, apreensão, insegurança, vulnerabilidade, além da necessidade de um reajuste comportamental e emocional para com o que está por vir e em como agir nas diversas situações cotidianas (Lemos \& Veríssimo, 2015; Barbosa, Balieiro \& Pettengill, 2012; Misquiatti, Brito, Ferreira \& Assumpção Júnior, 2015; von Kanel et al., 2019). No entanto, Dantas, Pontes, Assis e Collet (2012), contribuem às discussões, afirmando que em sua dinâmica cotidiana, a família buscará formas para se readaptar e enfrentar essa nova situação, a fim de resgatar o bem-estar de todos.

Outro aspecto a mencionar em relação à divisão do cuidado e das responsabilidades dos pais é fator imprescindível para atender às necessidades da criança, principalmente em famílias em que as alterações no desenvolvimento das crianças se fazem presente. Essa coparentalidade pode se dar na presença de um cônjuge ou de integrantes de uma rede de apoio social maior, como avós, tios e outros representantes do cuidado (Feinberg, 2002). A literatura tem mostrado que o nascimento de uma criança com alguma deficiência provoca impacto marcante na vida familiar, em especial na vida da mãe quando esta é cuidadora primária (Dantas, Pontes, Assis, \& Collet, 2012; Bastos \& Deslandes, 2008). Por outro lado, após o conhecimento do diagnóstico, e as etapas apresentadas na literatura, tais como: luto, choque, negação e aceitação vivenciados pelos pais, é necessário encontrar formas de adaptação às demandas emergentes da criança (Silva et al., 2010; Glat, 2012).

Os achados de Souza e Mariano (2018) mostram que a presença de cônjuge como fator positivo e, a ausência como fator negativo, não sejam necessariamente uma realidade, pois muitas vezes essas mulheres ainda se sentem sobrecarregadas com o trabalho diário, possuindo pouco apoio do outro. Isso se justifica por estudos como o de Sorj (2013), que discute o fato de que mulheres chefes de família trabalham menos nas tarefas domésticas do que as que tinham um cônjuge.

Desse modo, os achados dos estudos ratificam a necessidade de compreender as atividades relacionadas ao cuidado sob um viés generificado, buscando assimilar de uma forma mais abrangente as diferenças nas realidades de cuidadoras de crianças com desenvolvimento atípico, e como as relações de gênero em uma sociedade como a amazônica brasileira. O tópico a seguir busca atingir o objetivo do estudo, realizando tal análise.

\section{Por uma análise generificada da realidade na amazônia brasileira}

Uma compreensão interseccional, nos favorece $o$ entendimento, em relação à complexidade da realidade de cuidadoras de 
crianças com desenvolvimento atípico no contexto amazônico brasileiro. Isso é possível a partir da identificação de aspectos como gênero e classe como demarcadores analíticos desta realidade, que atravessam as relações existentes e marcam os comportamentos desse grupo social.

$\mathrm{Na}$ realidade amazônica, principalmente a de mulheres ribeirinhas (Santos, Nunes, Pontes, \& Silva, 2017), ainda prevalece a visão de homens como provedores e mulheres destinadas ao ambiente doméstico e de educação dos filhos. Tal afirmação é ratificada com os dados encontrados. De acordo com as Estatística de Gênero do Instituto Brasileiro de Geografia e Estatística (IBGE), na região Norte, enquanto as mulheres passam 17,2 horas por dia em média de horas dedicadas semanalmente aos cuidados de pessoas e/ou afazeres domésticos, os homens passam apenas 10,2 horas.

A partir desses dados, nota-se que os autores defendem um movimento de empoderamento dessas mulheres, principalmente quando beneficiárias de algum programa social como o Programa Bolsa Família (PBF), onde a transferência de renda assegurava certa estabilidade financeira, independência do marido-provedor, gerando maior controle das tomadas de decisão na família e na obtenção de recursos básicos para a casa e as crianças. Esses processos de empoderamento devem ser vistos, a partir de Silva e Martínez (2004), como multifocais, abrangendo desde as mulheres, de forma individual, até as coletividades existentes, pois, tais processos são dinâmicos e contribuem para o desenvolvimento humano, segundo os pressupostos de Bronfenbrenner (1989).

Somado a isso, têm-se a necessidade de considerar o contexto aos quais essas cuidadoras estão envolvidas. A oferta de serviços públicos de saúde, imprescindíveis para o acompanhamento de crianças com desenvolvimento atípico é abaixo do necessário para suprir a demanda apresentada (Afonso, 2016). Bronfenbrenner (2011) mostra em seus estudos, que um ambiente desfavorável em aspectos promotores de desenvolvimento (podemos acrescentar nessa descrição condições precárias de infraestrutura e mobilidade, um agravante considerando as distintas realidades das sub-regiões da Amazônia), irá gerar diversas consequências negativas para as pessoas ali inseridas, como as cuidadoras. Essa afirmação vem sendo revisitada por estudos que abordam as consequências negativas de atividades exercidas pelas cuidadoras (Barbosa et al., 2012; Dantas et al., 2012; Afonso, 2016; Dourado, França, Cardoso, da Costa Silva \& Pontes, 2017).

A pesquisa de Dourado, França, Cardoso, da Costa Silva e Pontes (2017) é relevante para o presente estudo, pois discutiu aspectos da coparentalidade de cuidadores de crianças com deficiência, no âmbito nortista, cujos resultados apontaram para a percepção de maior nível de suporte e exclusão do outro par coparental pelo cuidador secundário. Nessa investigação, os autores observaram que os cuidadores secundários só ajudavam em tarefas que condiziam ao cuidado da criança, cabendo ao cuidador primário, tarefas que exigiam mais esforço. Nesse estudo também foi encontrado que as cuidadoras principais, todas mulheres, obtiveram altos índices de conflito, representando uma insatisfação com a divisão de tarefas e justificada pela sobrecarga de papéis assumidos culturalmente.

Os estudos acima nos remetem à dicotomia público/privado, ainda perpetuada na realidade amazônica brasileira. Okin (2008) discute essa dicotomia, ao afirmar que essa separação entre o ambiente doméstico e o ambiente não doméstico contribui para a reprodução de comportamentos patriarcais, e tal fenômeno é reforçado pela ausência de análises generificadas a respeito da realidade estudada. Logo, ao excluir a compreensão de que o constructo gênero deve ser inserido nas análises dos diversos grupos sociais, exclui-se os produtos que esses constructos resultam em uma sociedade assimétrica, em matéria de desigualdade de gênero. 
Esse fato pode ser ratificado pela dificuldade na busca por dados estatísticos que possibilitem uma compreensão mais próxima da realidade acerca das realidades vivenciadas por cuidadoras de crianças com desenvolvimento atípico, pessoas essas que, ao se inserir de maneira relacional com o ambiente, são atravessadas por toda uma carga de ações e respostas de uma rede de contextos onde as atividades de cuidado ainda são vistas como "domínios" femininos. É a partir, primeiramente, do conhecimento dessas realidades, que proposições mais concretas podem ser pensadas, pois, como postula Bronfenbrenner (2011), os achados dos estudos empreendidos dentro da ciência, da forma a qual convencionamos nomear, devem ser traduzidas em indicadores para a construção de políticas públicas mais sensíveis para os grupos sociais alvos.

\section{Considerações finais}

O presente estudo buscou somar aos demais achados, que enfatizam ainda nos dias atuais, situações desafiadoras em mulheres cuidadoras mediante os desafios da discriminação de modo geral, confirmando assim, o viés e fortalecimento da generificação na realização de suas atividades, principalmente, em relação às crianças com desenvolvimento atípico, que demandam maiores cuidados, dadas as condições das práticas educativas parentais, bem como suas crenças que vão interferir no seu cotidiano.

Nota-se que os papeis de cuidados desempenhados são pensados e reforçados a partir de valores culturais que perpassam o tempo. Tais valores estão associados às categorias de gênero, raça e classe, como apontam os estudos interseccionais, o que aplicando à realidade amazônica, acaba por ser confirmado nas estatísticas de mulheres que exercem diversos papeis simultâneos, como os de mãe, de cuidadora, de provedora do lar, por exemplo. Esses papéis estão diretamente relacionados às atividades exercidas e as relações constituídas, o que, em uma perspectiva temporal, atravessa gerações e se perpetuam no decorrer da história.

Em diferentes trajetórias apresentadas e discutidas nos estudos, notaram-se registros, tais como: iniciativas de políticas públicas; perspectivas de superação das dificuldades de acessibilidade aos diversos espaços da sociedade; busca de fortalecimento para o processo identitário. Porém, cabe citar a pouca ênfase dada à necessidade de empoderamento das mães cuidadoras, nas práticas de cuidados de crianças com desenvolvimento atípico, que parecem requerer não apenas $\mathrm{o}$ apoio $\mathrm{e}$ sensibilidade da família, mas também tipos de intervenções que poderão organizar junto às famílias dessas crianças, buscando a equidade entre homens e mulheres.

A inserção de um trabalho de sensibilização e iniciativas de políticas públicas poderá garantir às mulheres cuidadoras, o desempenho de seus papéis parentais, sob o viés da responsividade, apoio e respeito em suas ações junto às crianças com desenvolvimento atípico e dessa forma, somar à qualidade de vida, a saúde, que melhorem essas práticas, no âmbito da sociedade.

\section{Referências}

Acton, G. J. (2002). Health-Promoting Self-

Care in Family Caregivers. Western

Journal of Nursing Research, 24(1), 73-86. doi: $10.1177 / 01939450222045716$

Afonso, T. (2016). Práticas de cuidado, redes de apoio e satisfação social de cuidadores primários de crianças com paralisia cerebral (Tese de doutorado). Universidade Federal do Pará, Belém
Barbosa, M. A. M., Balieiro, M. M. F. G., \& Pettengill, M. A. M. (2012). Cuidado centrado na família no contexto da criança com deficiência e sua família: uma análise reflexiva. Texto \& Contexto - Enfermagem, 21(1), 194-199. doi: https://dx.doi.org/10.1590/S0104$\underline{07072012000100022}$ 
Bastos, O. M. \& Deslandes, S. F. (2008). A experiência de ter um filho com deficiência mental: narrativas de mães. Cadernos de Saúde Pública, 24(9), 2141-2150. doi: 10.1590/S0102-311X2008000900020

Bronfenbrenner, U. (1989). Ecological systems theory. In: Vasta, R. (org.) Annals of Child Development. Greenwich: Jay. p. 187-249.

Bronfenbrenner, U. (2011). Bioecologia do Desenvolvimento Humano: tornando os seres humanos mais humanos. Porto Alegre: Artmed.

Bronfenbrenner, U. \& Morris, P. A. (2006). The bioecological model of human development. In W. Damon \& R. M. Lerner (Eds.), Handbook of child psychology: theoretical models of human developmental (Vol. 1, pp. 793-828). New York: John Wiley.

Butler, J. (2003). Problemas de gênero: feminismo e subversão da identidade. Rio de Janeiro: Civilização Brasileira.

Cairns, R. B., Elder, G. H., \& Costello, E. J. (Eds.). (1996). Developmental science. New York: Cambridge University Press.

Câmara, F. S., Martins, W. L. L., de Moura, M. L. N., Gadelha, E. C. M., Souza, L. L., \& Ferreira, J. M. S. (2016). Perfil do Cuidador de Pessoas com Deficiência. Revista Brasileira de Ciências da Saúde, 20(4), 269-276. doi:10.4034/RBCS.2016.20.04.02

Carvalho-Barreto, A. (2016). Paradigma Sistêmico no Desenvolvimento Humano e Familiar: a teoria bioecológica de Urie Bronfenbrenner. Psicologia em Revista, 22(2), 275-293. doi: 10.5752/P.16789523.2016V22N2P275.

Charmes, J. (2005). Femmes africaines, activités économiques et travail: de l'invisibilité à la reconnaissance. Revue Tiers Monde, $\mathrm{n}^{\circ}$ 182, avril, p. 255-281. DOI : $10.3917 / \mathrm{rtm} .182 .0255$

Collins, P. H. (2014). Interseccionality: a knowledge project for a decolonizing world. Comunicação ao colóquio internacional. Intersectionnalité et Colonialité: débats contemporains. Université Paris Diderot, 28 mar.
Connell, R. (2009). Gender in World Perspective. Cambridge, UK: Polity Press

Dantas, M. S. A., Pontes, J. F., Assis, W. D., \& Collet, N. (2012). Facilidades e dificuldades da família no cuidado à criança com paralisia cerebral. Revista Gaúcha de Enfermagem, 33(3), 73-80. doi: 10.1590/S1983-14472012000300010

Darling, N. (2007) Ecological systems theory: The person in the center of the circles. Research in Human Development, 4(3-4), 203-217. doi: 10.1080/15427600701663023

Dessen, M. A. \& Guedea, M. T. D. (2005). A ciência do desenvolvimento humano: ajustando o foco de análise. Paideia, 15(30), 11-21.

Dourado, C. M., França, I. L., Cardoso, V. S., da Costa Silva, S. S., \& Pontes, F. A. R. (2017). Coparenting in Caregivers of Children With Cerebral Palsy. Interpersona: An International Journal on Personal Relationships, 11(supp1), 35-46. doi: 10.5964/ijpr.v11isupp1.250

Faria, A. M. D. B. (2011). Crianças com transtorno do déficit de atenção e hiperatividade: um olhar sobre o cuidador primário (Dissertação de Mestrado). Faculdade de Filosofia, Ciências e Letras de Ribeirão Preto, Universidade de São Paulo, Ribeirão Preto. doi: 10.11606/D.59.2011.tde-23102013-134606.

Feinberg, M. E. (2002). Coparenting and the Transition to Parenthood: A Framework for Prevention. Clinical Child and Family Psychology Review, 5(3), 173-195. doi: 10.1023/a:1019695015110

Franco, V. (2016). Tornar-se pai/mãe de uma criança com transtornos graves do desenvolvimento. Educar em Revista, (59), 35-48. doi: $\underline{10.1590 / 0104-4060.44689}$

Furiati, L., \& Mayorga, C. (2016). Sem Lugar: A experiência de remoção de mulheres faveladas. Revista Polis e Psique, 6(2), 4569. Recuperado de http://pepsic.bvsalud.org/scielo.php?script= sci_arttext\&pid=S2238$152 X 2016000200004 \& \operatorname{lng}=\mathrm{pt} \& \mathrm{t} \operatorname{lng}=\mathrm{pt}$

Glat, R. (2012). Orientação familiar como estratégia facilitadora do desenvolvimento 
e inclusão de pessoas com necessidades especiais. In E. G. Mendes, M. A. Almeida (Org.), A pesquisa sobre inclusão escolar em suas múltiplas dimensões: teoria, política e formação (315-326). Marília: ABPEE

Henriques, C. R., Féres-Carneiro, T., \& Magalhães, A. S. (2006). Trabalho e família: o prolongamento da convivência familiar em questão. Paidéia (Ribeirão Preto), 16(35), 327-336. doi: 10.1590/S0103-863X2006000300004

Hirata, H. (2014). Gênero, classe e raça Interseccionalidade e consubstancialidade das relações sociais. Tempo Social, 26(1), 61-73. doi: $10.1590 / \mathrm{S} 0103-$ 20702014000100005

Kahana, E. \&; Young, R. (1990). Clarifying the caregiver paradigm: challenges for the future. In D. E. Biegel, \& A. Blum (eds.), Aging and caregiving: Theory, research and practice (76-97). Newbury Park, CA: Sage Publications

Kerner, I., \& Tavolari, B. (2012). Tudo é interseccional?: Sobre a relação entre racismo e sexismo. Novos estudos CEBRAP, (93), 45-58. doi: 10.1590/S010133002012000200005

Kotzampopoulou, I. (2015). Quality of life in families having children with disabilities: the parents' perspective (Dissertação de Mestrado). Universidade de Oslo, Noruega

Lemos, R. A., \& Veríssimo, M. L. Ó R. (2015). Development of premature children: caregivers' understanding according to the Bioecological Theory. Revista da Escola de Enfermagem da USP, 49(6), 898-906. doi: 10.1590/S0080-623420150000600004.

Magnusson, D., \& Cairns, R. B. (1996). Developmental science: Toward a unified framework. In R. B. Cairns, G. H. Elder, Jr., \& E. J. Costello (Eds.), Cambridge studies in social and emotional development. Developmental science (pp. 7-30). New York, NY, US: Cambridge University Press. http://dx.doi.org/10.1017/CBO9780511571 $\underline{114.003}$
Miranda, S. F. (2016). Da base da pirâmide social à "elite" do sistema: um estudo de caso sobre as diversas incursões de uma mulher negra, nordestina e militante.

Pesquisas e Práticas Psicossociais. 11(1), 100-117. Recuperado de

http://pepsic.bvsalud.org/scielo.php?script= sci_arttext\&pid=S180989082016000100009\&lng=pt\&tlng=pt

Misquiatti, A. R. N., Brito, M. C., Ferreira, F. T. S. \& Assumpção Júnior, F. B. (2015). Sobrecarga familiar e crianças com transtornos do espectro do autismo: perspectiva dos cuidadores. Revista CEFAC, 17(1), 192-200. doi: $\underline{10.1590 / 1982-0216201520413}$

Monteiro, A. M. (2001). Avanços no estudo da conjugalidade: os casais de dupla carreira. Psicologia: Ciência e Profissão, 21(3), 1019. doi: $10.1590 /$ S141498932001000300003

Morris, M. (2001). Gender-Sensitive Home and Community Care and Caregiving Research: a synthesis paper. Women Health Bureau of the Government of Canada.

Mthembu, T. G., Brown, Z., Cupido, A., Razack, G., \& Wassung, D. (2016). Family caregivers' perceptions and experiences regarding caring for older adults with chronic diseases. South African Journal of Occupational Therapy, 46(1), 83-88. doi: 10.17159/2310-3833/2016/v46n1a15

Okin, S. M. (2008). Gênero, o público e o privado. Revista Estudos Feministas, 16(2), 305-332. doi: 10.1590/S0104026X2008000200002

Oliveira, M. F. S., Silva, M. B. M., Frota, M. A., Pinto, J. M. S., Frota, L. M. C., \& Sá. F. E. (2008). Qualidade de Vida do Cuidador de Crianças com Paralisia Cerebral. RBPS, 21(4), 275-280.

Pinto, N. M. A. (2013). As redes de apoio social e as relações de trabalho de mulheres em comunidades rurais do Maranhão e ribeirinhas da região amazônica (Tese de Doutorado). Programa de Pós-Graduação em Teoria e Pesquisa do Comportamento, Universidade Federal do Pará, Belém. Recuperado de 
http://ppgtpc.propesp.ufpa.br/ARQUIVOS/t eses/Neuzeli\%20Pinto\%202013.pdf

Poletto, M., \& Koller, S. H. (2008). Contextos ecológicos: promotores de resiliência, fatores de risco e de proteção. Estudos de Psicologia (Campinas), 25(3), 405-416. doi: 10.1590/S0103-166X2008000300009

Pontes de Lima, A., \& Oliveira Lima, A. (2012). Perfil do desenvolvimento neuropsicomotor e aspectos familiares de crianças institucionalizadas na cidade do Recife. Revista CES Psicologia, 5(1), 11-24.

Recuperado de http://revistas.ces.edu.co/index.php/psicolo gia/article/view/2051

Santos, T. M., Nunes, B., Pontes, F. A. R., \& Silva, S. S. C. (2017). Female Empowerment of Amazonian Riverine Beneficiaries of the Bolsa Família Program. Interpersona: An International Journal on Personal Relationships, 11(supp1), 22-34. doi: 10.5964/ijpr.v11isupp1.249

Sena, E., Souza, M., \& Andrade, L. (2016). Percepção de cuidadores de pessoas com Doença de Alzheimer sobre suas perspectivas de futuro. Revista Kairós: Gerontologia, 19(3), 151-168. Recuperado de

https://revistas.pucsp.br/kairos/article/view/ 32369/22401

Silva, C., \& Martínez, M. L. (2004).

Empoderamiento: Proceso, Nivel y Contexto. PYSKHE, 13(1), 29-39. doi: 10.4067/S0718-22282004000200003

Silva, S. S. C., Pontes, F. A. R., Lima, L. C., \& Maluschke, J. B. (2010). Rede social e papéis de gênero de casais ribeirinhos de uma comunidade amazônica. Psicologia: Teoria e Pesquisa, 26(4), 605-612. doi: 10.1590/S0102-37722010000400004

Silva, T. C. O., Barros, V. F., \& Hora, E. C. (2011). Experiência de ser um cuidador familiar no câncer infantil. Rev. Rene, 12(3), 526-531. Recuperado de http://periodicos.ufc.br/rene/article/view/42 $\underline{76}$
Sorj, B. (2013). Arenas de cuidado nas interseções entre gênero e classe social no Brasil. Cadernos de Pesquisa, 43(149), 478-491. doi: 10.1590/S010015742013000200006

Sorj, B. (2014). Socialização do cuidado e desigualdades sociais. Tempo Social, revista de sociologia da USP. v. $26, \mathrm{n} .1, \mathrm{p}$. 123-128.

Souza, M. F., \& Mariano, S. (2018). Percepções de Cuidado e Práticas de Gênero de Mulheres em Situação de Pobreza a Partir de um Recorte Geracional. Mediações - Revista de Ciências Sociais, 23(3), 164-194. doi: 10.5433/21766665.2018v23n3p164

Swinkels, J., van Tilburg, T., Verbakel, E., \& Van Groenou, M. B. (2017). Explaining Gender Gap in the Caregiving Burden of Parent Caregivers. The Journals of Gerontology: Series B, 74(2), 309-317. doi: $10.1093 /$ geronb/gbx036.

Wagner, A., Predebon, J., Mosmann, C., \& Verza, F. (2005). Compartilhar tarefas? Papéis e funções de pai e mãe na família contemporânea. Psicologia: Teoria e Pesquisa, 21(2), 181-186. doi: 10.1590/S0102-37722005000200008

Van Keer, I., Colla, S., Van Leeuwen, K., Vlaskamp, C., Ceulemans, E., Hoppenbrouwers, K.,... Maes, B. (2017). Exploring parental behavior and child interactive engagement: A study on children with a significant cognitive and motor developmental delay. Research in Developmental Disabilities, 64, 131-142. doi: $10.1016 /$ j.ridd.2017.04.002

Von Kanel, R., Mausbach, B. T., Dimsdale, J. E., Ziegler, M. G., Mills, P. J., Allison, M. A.,... \& Grant, I. (2019). Refining caregiver vulnerability for clinical practice: determinants of self-rated health in spousal dementia caregivers. BMG Geriatrics, 19(18), 1-11. doi: 10.1186/s12877-019$\underline{1033-2}$ 


\section{Dados sobre os autores:}

- Matheus dos Santos da Silveira: Graduado em Relações Internacionais pela Universidade da Amazônia (2018), Mestrando em Teoria e Pesquisa do Comportamento pela Universidade Federal do Pará (2019-Atual), graduando em Psicologia pela Universidade Federal do Pará (2015-Atual). Desenvolve pesquisas no Laboratório de Ecologia do Desenvolvimento (LEDUFPA) em temas como: Psicologia do Desenvolvimento Humano e Psicologia Social.

- Yasmin Borges Farias: Graduanda em Psicologia na Universidade Federal do Pará (2015Atual), bolsista do CNPq no Laboratório de Ecologia e Desenvolvimento (LED) do Núcleo de Teoria e Pesquisa do Comportamento (NTPC). Atua principalmente nos seguintes temas: pobreza, desenvolvimento, família e coparentalidade. Possui interesse nas áreas de Psicologia do Desenvolvimento Humano, Psicologia Clínica e Feminização da Pobreza.

- Ruth Daisy Capistrano de Souza: Graduada em Pedagogia pela União das Escolas Superiores do Pará (1988), mestra e doutora em Teoria e Pesquisa do Comportamento - Área: Psicologia, pela Universidade Federal do Pará (2004/2008), realiza estágio pós- doutoral em Ecoetologia do Desenvolvimento (2016-Atual) na Universidade Federal do Pará. Experiência na área de Educação, Educação Especial e diversidade humana, Psicologia (ênfase em Processos de Aprendizagem) atuando principalmente nos seguintes temas: História da Educação, Planejamento Educacional, Currículo, Surdez, TDAH, Deficiência Múltipla, Inclusão, Ensino e Aprendizagem, Família e Alteração em Desenvolvimento humano. 